REVIEWS

\title{
The Effect of Charge Display on Cost of Care and Physician Practice Behaviors: A Systematic Review
}

\author{
Celine Goetz, MD' , Stephen R. Rotman, MD' , George Hartoularos ${ }^{3}$, and Tara F. Bishop, MD, MPH 5.6 \\ 'Division of Hospital Medicine, Department of Medicine, Mount Sinai Hospital, New York, NY, USA; ${ }^{2}$ Division of Gastroenterology, Brigham and \\ Women's Hospital, Boston, MA, USA; ${ }^{3}$ Department of Chemical Engineering, Massachusetts Institute of Technology, Cambridge, MA, USA; ${ }^{4}$ Weill \\ Cornell Medical College Medical Library, New York, NY, USA ; ${ }^{5}$ Division of Outcomes and Effectiveness, Department of Public Health, Weill Cornell \\ Medical College, New York, NY, USA; ${ }^{6}$ Division of General Internal Medicine, Department of Medicine, Weill Cornell Medical College, New York, NY, USA.
}

BACKGROUND: While studies have been published in the last 30 years that examine the effect of charge display during physician decision-making, no analysis or synthesis of these studies has been conducted.

OBJECTIVE: We aimed to determine the type and quality of charge display studies that have been published; to synthe-size this information in the form of a literature review.

METHODS: English-language articles published between 1982 and 2013 were identified using MEDLINE, Web of Knowledge, ABI-Inform, and Academic Search Premier. Article titles, abstracts, and text were reviewed for relevancy by two authors. Data were then extracted and subsequently synthesized and analyzed.

RESULTS: Seventeen articles were identified that fell into two topic categories: the effect of charge display on radiology and laboratory test ordering versus on medication choice. Seven articles were randomized controlled trials, eight were pre-intervention vs. post-intervention studies, and two interventions had a concurrent control and intervention groups, but were not randomized. Twelve studies were conducted in a clinical environment, whereas five were survey studies. Of the nine clinically based interventions that examined test ordering, seven had statistically significant reductions in cost and/or the number of tests ordered. Two of the three clinical studies looking at medication expenditures found significant reductions in cost. In the survey studies, physicians consistently chose fewer tests or lower cost options in the theoretical scenarios presented.

CONCLUSIONS: In the majority of studies, charge information changed ordering and prescribing behavior.

KEY WORDS: charge display; physician behavior; costs of care.

$\mathrm{J}$ Gen Intern Med 30(6):835-42

DOI: $10.1007 /$ s11606-015-3226-5

(c) Society of General Internal Medicine 2015

\section{INTRODUCTION}

The United States consistently outspends other industrialized countries on health care, but with lower health care outcomes. ${ }^{1}$

Helen-Ann Brown Epstein has been retired.

Received September 14, 2014

Revised December 18, 2014

Accepted January 28, 2015

Published online February 18, 2015
Healthcare costs in the United States were $17.9 \%$ of the gross domestic product (GDP) in 2012 according to the World Bank - the highest GPD percentage spent on health care in the world. ${ }^{2}$ At the same time, US life expectancy lags behind other developed nations. ${ }^{1}$ One contributing factor to rising health care costs is that physicians rarely know the charges of the services, tests, and procedures they order or perform. ${ }^{3,4}$ This disconnect between the trend in rising health care costs and physician lack of knowledge about the financial impact of their management decisions leads to an obvious concept for an intervention: provide a currency amount for the intervention in question for physicians when they are deciding what tests to order or what medications to prescribe. Yet, this has hardly become commonplace practice in the United States. ${ }^{5-8}$

There have, however, been a number of studies that have tested the hypothesis that price information reduces ordering and costs, but there has been no synthesis of these studies. This information could be informative to policymakers, patient advocates, insurance companies, hospitals, and medical groups, who are all trying to find ways to reduce overuse and control costs.

The purpose of this systematic review was to determine the type of charge display studies that have been published, the quality of these studies, and their findings. We wanted to synthesize this information in the form of a literature review. We set out to identify studies where the intervention provided medical practitioners with a currency amount that reflected the charge of what they were ordering in real time, and then analyzed the differences in ordering behavior. We chose to narrow in on reviewing interventions specifically looking at real time charge display and its effect on physician decisions, rather than the broader topic of performance feedback, which was thoroughly explored in a recent Cochrane Review by Ivers et al., ${ }^{9}$ as well as more general literature review by Axt-Adam et al. in the early 1990s. ${ }^{10}$

We would like to comment on terminology at this juncture. The terms "price," "cost," "charge," and "fee" are often used interchangeably in this literature, even though there are nuanced differences to these terms. All interventions we examined included a currency value that physicians could incorporate into their management decisions. The source of this currency value was not consistently disclosed, as we will discuss 
later. In every health system, there are many layers to how the costs are generated and how services are paid for, thereby generating prices, fees, charges, etc. This systematic review did not attempt to reconcile these differences, but to assess various interventions with aforementioned intention. Throughout this review, we refer to currency amounts displayed using the same term as the authors of the paper did in their description. For our discussion, we chose the term "charge display" to discuss the concept as it pertains to physician decision-making.

\section{METHODS}

Design, Data Sources, and Search Criteria. We performed a systematic review of English-language articles published between 1982 and October 2013 using MEDLINE, Web of Knowledge, ABI-Inform, and Academic Search Premier, the details of which are outlined in Figure 1. The search strategy was developed by two of the authors (C.G. and H.A.B.E.). Search terms included medical descriptors, financial terms, behavior descriptors, and medical action as detailed in Table 1. We also manually searched reference lists in relevant articles.

Study Selection. We included articles that studied the effect of charge display interventions (including educational interventions) on the use of services, cost of care, or changes in physician decisions. The intervention had to provide charge data in "real time"-meaning that a currency value was displaced to the provider at the time of ordering. We included studies that had both a concurrent comparison group and those that used a pre-intervention vs. post-intervention design with no concurrent comparison group. We only included studies that provided quantitative results. We did not include studies where the outcome was change in attitudes, but did include studies where the outcome was change in case-based decisions. One reviewer (C.G.) assessed titles for relevance. Two reviewers (C.G. and S.R.R.) assessed selected abstracts for relevance and full articles for inclusion. When the reviewers disagreed, an additional reviewer (T.F.B.) resolved the discrepancy.

Data Extraction. Two authors (C.G. and G.H.) extracted the following data from selected articles: study design, setting, type of intervention, type of participants, number of participants, bias considerations, type of outcome measures, and results. These data are organized in Table 2.

Data Synthesis and Analysis. We grouped studies into two categories: 1) laboratory and radiology test ordering, 2) medication choices. For each study, we focused on three types of outcomes: 1) use of specific medical services or treatments, 2) cost of care, and 3) physician decisions. We were unable to perform a meta-analysis, because the studies were too heterogeneous.

\section{RESULTS}

Of the 4,513 articles identified through electronic search (search terms are outlined in Table 1), 71 articles were selected after title review, and from those articles, eight articles were selected by two reviewers after full article review. We identified nine more articles through reference review, for a total of 17 articles (Fig. 1).

Twelve studies were conducted in a clinical environment ${ }^{11-22}$ while five were survey or simulation studies (i.e., studies that asked physicians how they might behave in a clinical setting). ${ }^{23-27}$

Of the seventeen studies, seven were randomized controlled trials, ${ }^{11,12,14,19,23,24,26}$ eight were pre-intervention vs. postintervention studies, ${ }^{15,16,18,20-22,25,27}$ and two had a concurrent control and intervention groups, but were not randomized. ${ }^{13,17}$ Eleven studies examined physician ordering of laboratory or radiology testing, ${ }^{11-19,23,24}$ while six looked at medication choice. ${ }^{20-22,25-27}$ The details of the study design, study size, bias considerations, and follow-up period for the included studies are summarized in Table 2.

\section{Interventions in a Clinical Setting}

Effects on Radiology and Laboratory Test Ordering. There were a total of nine papers in this category: four randomized controlled trials, ${ }^{11,12,14,19}$ two non-randomized controlled tri$\mathrm{al},{ }^{13,17}$ and three pre-intervention vs. post-intervention studies ${ }^{15,16,18}$ that looked at test ordering. Four interventions were conducted in inpatient wards ${ }^{11-14}$ two were conducted in emergency department, ${ }^{15,17}$ two were in intensive care units, ${ }^{16,18}$ and one was in an internal medicine outpatient clinic. ${ }^{19}$ Six were conducted in the United States ${ }^{11,12,14-16,19}$; studies were also conducted in South Africa, ${ }^{13}$ Sweden, ${ }^{17}$ and France. ${ }^{18}$ Two studies came from the pediatric literature. ${ }^{15,16}$

The clinical interventions themselves included four electronic medical record (EMR) ${ }^{11,12,14,19}$ and five paper-based interventions. ${ }^{13,15-18}$ The EMR interventions very similar: the window for a patient's orders included the charge amounts. ${ }^{12,14,19}$ Bates et al. had a "cash register" component that totaled the charges. ${ }^{11}$ Among the paper-based methods, Hampers et al. and Seguin et al. placed charges next to the items ordered on paper order forms in a pediatric $\mathrm{ED}^{15}$ and in a French intensive care unit, ${ }^{18}$ respectively. Sachedeva et al. placed itemized charges from the prior day's test charges every morning where orders were subsequently placed. ${ }^{16}$ Ellemdin et al. gave physicians a pocket-sized brochure with laboratory costs; physicians then had to write that amount on the order requisition. ${ }^{13}$ Schilling distributed price lists via email to physicians and then had this list displayed at the physicians' workstations. ${ }^{17}$

Only four studies reported the sources of displayed charges. Two studies stated that the currency amounts reflected what the clinic or hospital charged to the insurer or to the patient if the patient did not have insurance, ${ }^{11,19}$ and two other studies used the Medicare allowable fee for the test. ${ }^{12,14}$

Three studies were designed with a quality metric in place. Hampers et al.'s design included follow-up phone calls to see if the patient had been medically re-evaluated and to assess 


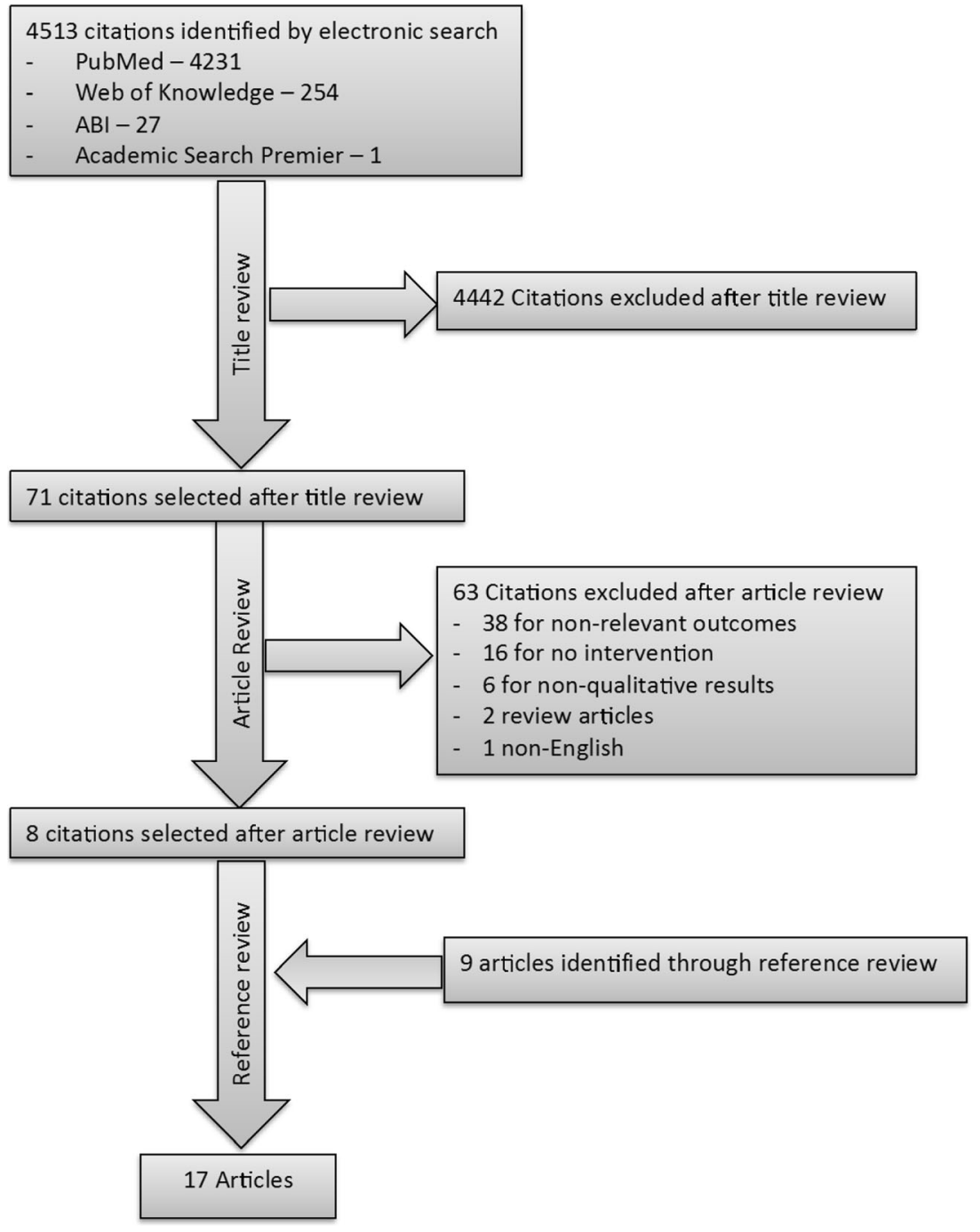

Figure 1. Review process.

satisfaction with care. Differences in control vs. intervention groups on both accounts were not statically significant. ${ }^{15}$ Sachdeva et al. collected data on occurrences of pediatric ICU-related complications during the control and intervention periods to measure quality of care. Length of stay in the ICU and mortality between the two groups were not statistically significant. ${ }^{16}$ Tierney et al. reviewed patients' computer records for 26 weeks following the intervention period to compare rates of hospitalization, emergency room visits, and outpatient visits. No significant differences were found. ${ }^{19}$ The other six studies did not report a quality metric.

Of the nine clinically based interventions that examined test ordering, seven had statistically significant reductions in cost and/or the number of tests ordered. These results are fully detailed in Table 2. Feldman et al. reported decreases in number of tests ordered in both the intervention and control arms compared to preintervention rates; still, the difference in decreases in the

Table 1. Search Terms

\begin{tabular}{lll}
\hline \hline Medical descriptors & Economic terms & Behavior descriptors \\
\hline Doctor & Pric* & Dedical actions \\
Physician & Fee* & Deci* \\
Medic* & Charge* & Influen* \\
Hospital & Budget & Bias \\
Lab* & Cost & Persua* \\
Radiology & & Convince \\
Imaging & & Habit \\
& & Appropriate* \\
& & Studeppropriate* \\
& & Aware* \\
& Transparenc* \\
\hline
\end{tabular}


Table 2. Summary of Evidence

\begin{tabular}{|c|c|c|c|c|}
\hline Article citation & Study details & Bias considerations & Intervention & Results \\
\hline \multicolumn{5}{|c|}{ Articles on Lab/Radiology Ordering } \\
\hline $\begin{array}{l}\text { Bates, D. et al. Does } \\
\text { the Computerized } \\
\text { Display of Charges } \\
\text { Affect Inpatient Ancillary } \\
\text { Test Utilization? Arch } \\
\text { Intern Med. 1997; } \\
\text { 157: 2501-2508 }\end{array}$ & $\begin{array}{l}\text { - Randomized } \\
\text { Controlled } \\
\text { design } \\
\text { - Lab test subjects: } \\
\text { 3,536 vs. } 3,554 \\
\text { inpatient orders; } \\
\text { radiology: } 8,728 \\
\text { vs. } 8,653 \text { inpatient } \\
\text { orders }\end{array}$ & $\begin{array}{l}\text { - Randomization } \\
\text { performed using } \\
\text { internal identification } \\
\text { number not available } \\
\text { to providers } \\
\text { - Labs sent directly } \\
\text { to lab via envelope } \\
\text { were not captured. } \\
\text { Only } 53 \% \text { of lab } \\
\text { tests had an associated } \\
\text { computer order. } 74 \% \\
\text { of radiology testing } \\
\text { had a computer order. }\end{array}$ & $\begin{array}{l}\text { The cost for each test } \\
\text { ordered was displayed } \\
\text { by the specific test, } \\
\text { as well as the total for } \\
\text { the ordering session in } \\
\text { a "cash register" } \\
\text { window below the } \\
\text { tests list. }\end{array}$ & $\begin{array}{l}4.5 \% \text { decrease in clinical } \\
\text { laboratory tests ordered; } \\
\text { total charges were } 4.2 \% \\
\text { lower. Radiology ordering } \\
\text { was reported as practically } \\
\text { identical in control and } \\
\text { experimental periods. } \\
\text { Cost Reduction: Not } \\
\text { statistically significant } \\
\text { Decrease in number of tests } \\
\text { ordered not statistically } \\
\text { significant }\end{array}$ \\
\hline $\begin{array}{l}\text { Cummings, K. M. et al. } \\
\text { The Effects of Price } \\
\text { Information on Physicians' } \\
\text { Test-Ordering Behavior. } \\
\text { Medical Care. 1982; } 20 \\
\text { (3): 293-301. }\end{array}$ & $\begin{array}{l}\text { - Randomized } \\
\text { Controlled design } \\
\text { - Survey study of } \\
36 \text { family medicine } \\
\text { residents and } 23 \\
\text { faculty in US }\end{array}$ & $\begin{array}{l}\text { - No description of } \\
\text { randomization process } \\
\text { - } 59 \text { of } 67 \text { subjects } \\
\text { contacted } \\
\text { participated in the study } \\
\text { ( } 88 \% \text { response rate) }\end{array}$ & $\begin{array}{l}\text { Participants reviewed } \\
\text { four cases in which } \\
\text { certain diagnoses were } \\
\text { intended, and then } \\
\text { asked to order tests. } \\
\text { One group used a test } \\
\text { order forms with } \\
\text { prices, the other } \\
\text { without. }\end{array}$ & $\begin{array}{l}\text { The price-information group } \\
\text { ordered } 30.8 \% \text { fewer tests } \\
\text { per patient than did the } \\
\text { control group. } \\
\text { Cost Reduction: Statistically } \\
\text { significant } \\
\text { Decrease in number of tests } \\
\text { ordered not consistently } \\
\text { statistically significant }\end{array}$ \\
\hline $\begin{array}{l}\text { Durand, DJ et al. } \\
\text { Provider Cost Transparency } \\
\text { Alone Has No Impact } \\
\text { on Inpatient Imaging } \\
\text { Utilizations. J Am } \\
\text { Coll Radiol 2013; } \\
\text { 10: } 108-113 .\end{array}$ & $\begin{array}{l}\text { - Randomized } \\
\text { Controlled design } \\
\text { - } 5 \text { vs. } 5 \text { radiology } \\
\text { tests randomized } \\
\text { to display vs. } \\
\text { control }\end{array}$ & $\begin{array}{l}\text { - Randomization was } \\
\text { performed using } \\
\text { web-based software }\end{array}$ & $\begin{array}{l}\text { The difference in ordering } \\
\text { of radiology tests } \\
\text { randomized to display vs. } \\
\text { blind ordering over } 6 \\
\text { months, compared to } \\
\text { similar 6-month } \\
\text { baseline test-ordering data. }\end{array}$ & $\begin{array}{l}\text { Change in utilization was } \\
\text { not significant } \\
\text { between the two groups. }\end{array}$ \\
\hline $\begin{array}{l}\text { Ellemdin, S et al. } \\
\text { Providing Clinicians } \\
\text { with Information } \\
\text { on Laboratory Test } \\
\text { Costs Leads to Reduction } \\
\text { in Hospital Expenditure. } \\
\text { S Afr Med J. 2011; 201: } \\
746-748 .\end{array}$ & $\begin{array}{l}\text { - Non-Randomized } \\
\text { Controlled design } \\
\text { - } 463 \text { vs. } 434 \\
\text { Inpatient orders on } \\
\text { wards of South } \\
\text { African hospital }\end{array}$ & $\begin{array}{l}\text { - Control and } \\
\text { intervention groups } \\
\text { were matched in } \\
\text { terms of experience } \\
\text { - } 100 \% \text { compliance } \\
\text { with intervention }\end{array}$ & $\begin{array}{l}\text { Laboratory test costs were } \\
\text { provided to clinicians as } \\
\text { a pocket-sized brochure. } \\
\text { They were asked to write } \\
\text { in the cost of every test } \\
\text { ordered on the laboratory } \\
\text { test request form. }\end{array}$ & $\begin{array}{l}27 \% \text { reduction in mean cost } \\
\text { per patient admitted and } 36 \% \\
\text { reduction in mean cost } \\
\text { per day of entire intervention group. } \\
\text { Cost Reduction: Statistically } \\
\text { significant } \\
\text { Change in number of tests } \\
\text { ordered not reported }\end{array}$ \\
\hline $\begin{array}{l}\text { Feldman, L et al. Impact } \\
\text { of Providing Fee Data on } \\
\text { Laboratory Test Ordering. } \\
\text { JAMA Intern Med. 2013; } \\
\text { 173(10): 903-908 }\end{array}$ & $\begin{array}{l}\text { - Randomized } \\
\text { Controlled design } \\
\text { - } 61 \text { lab tests } \\
\text { randomized to } \\
\text { display vs. blind }\end{array}$ & $\begin{array}{l}\text { - No description of } \\
\text { randomization } \\
\text { process } \\
\text { - Providers were unaware } \\
\text { that displayed fees were } \\
\text { part of study unless } \\
\text { research team was } \\
\text { specifically questioned }\end{array}$ & $\begin{array}{l}\text { The difference in ordering } \\
\text { of } 61 \text { lab tests randomized } \\
\text { to display vs. blind } \\
\text { ordering over } 6 \text { months, } \\
\text { compared to similar } \\
\text { 6-month baseline } \\
\text { test-ordering data. }\end{array}$ & $\begin{array}{l}9.1 \% \text { decrease of number } \\
\text { of tests ordered from baseline } \\
\text { period in active arm; control } \\
\text { arm saw } 5.1 \% \text { increase. Total } \\
\text { fees for laboratory testing dropped } \\
10.1 \% \text { in active arm. } \\
\text { Cost Reduction: statistically } \\
\text { significant } \\
\text { Decrease in number of tests } \\
\text { ordered: statistically significant }\end{array}$ \\
\hline $\begin{array}{l}\text { Hampers, L.C. et al. The } \\
\text { Effect of Price Information } \\
\text { on Test-ordering Behavior } \\
\text { and Patient Outcomes in a } \\
\text { Pediatric Emergency } \\
\text { Department. Pediatrics. } \\
\text { 1999; 103: 877-882. }\end{array}$ & $\begin{array}{l}\text { - Pre-Intervention, } \\
\text { Post-Intervention } \\
\text { design } \\
\text { - 2,467 vs. } 2,414 \\
\text { orders in Pediatric } \\
\text { ER in US }\end{array}$ & $\begin{array}{l}\text { - Demographic } \\
\text { differences in control } \\
\text { and intervention } \\
\text { periods are not } \\
\text { statistically } \\
\text { significant. } \\
\text { - Control period and } \\
\text { intervention period } \\
\text { were seasonally } \\
\text { different }\end{array}$ & $\begin{array}{l}\text { During the intervention } \\
\text { period, emergency room } \\
\text { physicians used test order } \\
\text { forms that listed the } \\
\text { standard hospital charge } \\
\text { for each test. }\end{array}$ & $\begin{array}{l}\text { Charges for tests in the } \\
\text { intervention period were } \\
27 \% \text { less than charges } \\
\text { in the control period, } \\
\text { without compromising } \\
\text { quality of care, as determined } \\
\text { by a follow phone call. } \\
\text { Cost Reduction: Statistically } \\
\text { significant } \\
\text { Decrease in number of tests } \\
\text { ordered statistically } \\
\text { significant }\end{array}$ \\
\hline $\begin{array}{l}\text { Rudy, D. W. et al. } \\
\text { A Pilot Study Assessing } \\
\text { the Influence of Charge } \\
\text { Data and Group Process } \\
\text { on Diagnostic Test } \\
\text { Ordering by Residents. } \\
\text { Acad Med. } 2001 \text {; } \\
\text { 76: } 635-637 .\end{array}$ & $\begin{array}{l}\text { - Randomized } \\
\text { Controlled design } \\
\text { - Survey of American } \\
23 \text { IM residents }\end{array}$ & $\begin{array}{l}\text { - No description of } \\
\text { randomization } \\
\text { process } \\
\text { or participation rate }\end{array}$ & $\begin{array}{l}\text { At a resident workshop, } \\
\text { participants were given a } \\
\text { hypothetical case and split } \\
\text { into three groups and } \\
\text { ordered lab tests: (A) } \\
\text { given charge data before } \\
\text { ordering, (B) given } \\
\text { charge data after ordering, } \\
\text { and (C) not given charge } \\
\text { data at all. }\end{array}$ & $\begin{array}{l}\text { Residents who had charge } \\
\text { data access spent considerably } \\
\text { less }(\$ 1,297 \text { vs. } \$ 2,205) \text { but } \\
\text { had lower appropriateness scores. } \\
\text { Cost Reduction: Statistically } \\
\text { significant } \\
\text { Change in number of tests } \\
\text { ordered not reported }\end{array}$ \\
\hline
\end{tabular}


Table 2. (continued)

\begin{tabular}{|c|c|c|c|c|}
\hline Article citation & Study details & Bias considerations & Intervention & Results \\
\hline $\begin{array}{l}\text { Sachdeva, R. C. et al. } \\
\text { Effects of Availability } \\
\text { of Patient-related } \\
\text { Charges on Practice } \\
\text { Patterns and Cost } \\
\text { Containment in the } \\
\text { Pediatric Intensive } \\
\text { Care Unit. Crit Care } \\
\text { Med. 1996; 24: } \\
\text { 501-506. }\end{array}$ & $\begin{array}{l}\text { - Pre-Intervention, } \\
\text { Post-Intervention } \\
\text { design } \\
\text { - } 598 \text { patients in } \\
\text { Pediatric ICU in US }\end{array}$ & $\begin{array}{l}\text { - Practitioners were } \\
\text { aware of intervention, } \\
\text { but exact nature of } \\
\text { study was not disclosed } \\
\text { to minimize bias } \\
\text { - Control period and } \\
\text { intervention period } \\
\text { were seasonally } \\
\text { different }\end{array}$ & $\begin{array}{l}\text { Daily patient-related } \\
\text { itemized charges for } \\
\text { laboratory and radiology } \\
\text { tests that had been ordered } \\
\text { in the previous } 24-\mathrm{h} \text { time } \\
\text { period were displayed in } \\
\text { the patient's chart. }\end{array}$ & $\begin{array}{l}\text { Lab and pharmacy charges } \\
\text { decreased. The study } \\
\text { adjusted for severity of } \\
\text { illness and intensity of } \\
\text { medical and nursing } \\
\text { interventions. } \\
\text { Cost Reduction: Statistically } \\
\text { significant } \\
\text { Change in number of tests } \\
\text { ordered not reported }\end{array}$ \\
\hline $\begin{array}{l}\text { Schiling, U. M. Cutting Costs: } \\
\text { the Impact } \\
\text { of Price Lists on the } \\
\text { Cost Development } \\
\text { at the Emergency } \\
\text { Department. Eur J } \\
\text { Emerg Med. } \\
\text { 2010; 17: 337-339. }\end{array}$ & $\begin{array}{l}\text { - Non-randomized } \\
\text { Controlled design } \\
\text { - 6,131 orders in } \\
\text { Swedish ER }\end{array}$ & $\begin{array}{l}\text { - Two different } \\
\text { emergency room orders } \\
\text { were compared } \\
\text { (Orthopedic vs. } \\
\text { Medical) } \\
\text { - Physicians were not } \\
\text { specifically made } \\
\text { aware of the study }\end{array}$ & $\begin{array}{l}\text { Price lists of the } 91 \text { most } \\
\text { common lab tests and } 39 \\
\text { most common radiological } \\
\text { tests were distributed to } \\
\text { Medicine physicians via } \\
\text { e-mail and were displayed } \\
\text { at the medical working } \\
\text { stations. Orthopedic ER } \\
\text { served as control. }\end{array}$ & $\begin{array}{l}\text { In the experimental group, } \\
\text { lab analysis costs decreased } \\
\text { by } 21.4 \% \text { (vs. decrease of } 9 \\
\% \text { and radiology costs by } \\
20.59 \% \text { (vs. increase of } 5.4 \\
\% \text { ). The total number of } \\
\text { radiological investigations } \\
\text { decreased by } 4.6 \% \\
\text { (control) vs. } 6.8 \% \\
\text { (experimental) } \\
\text { Cost Reduction: Statistically } \\
\text { significant } \\
\text { Decrease in number of tests } \\
\text { ordered only reported for } \\
\text { radiology and was not } \\
\text { statistically significant }\end{array}$ \\
\hline $\begin{array}{l}\text { Seguin, P. et al. } \\
\text { Effects of Price } \\
\text { Information on } \\
\text { Test Ordering in an } \\
\text { Intensive Care Unit. } \\
\text { Intensive Care Med. } \\
\text { 2002; 28: 332-335. }\end{array}$ & $\begin{array}{l}\text { - Pre-Intervention, } \\
\text { Post-Intervention } \\
\text { design } \\
\text { - Orders for } 287 \\
\text { patients French } \\
\text { ICU }\end{array}$ & $\begin{array}{l}\text { - Control and } \\
\text { interventional } \\
\text { periods were } \\
\text { seasonally similar } \\
\text { - Data collected by } \\
\text { physicians who did } \\
\text { not practice during } \\
\text { the study duration } \\
\text { - Medical staff was } \\
\text { not notified that a } \\
\text { study was in process }\end{array}$ & $\begin{array}{l}\text { Prices for the seven } \\
\text { most frequently ordered } \\
\text { tests were included on } \\
\text { the lab test ordering } \\
\text { form during the } \\
\text { intervention period. }\end{array}$ & $\begin{array}{l}\text { The total cost reduction was } \\
\text { significant, but significance } \\
\text { was not achieved for } \\
\text { all individual tests. } \\
\text { Cost Reduction: Statistically } \\
\text { significant } \\
\text { Decrease in number of tests } \\
\text { ordered not consistently } \\
\text { statistically significant }\end{array}$ \\
\hline $\begin{array}{l}\text { Tierney, W. M. et al. } \\
\text { The Effect on Test } \\
\text { Ordering of Informing } \\
\text { Physicians of the } \\
\text { Charges for } \\
\text { Outpatient Diagnostic } \\
\text { Tests. N Engl J Med. } \\
\text { 1990; 322: 1499-1504 }\end{array}$ & $\begin{array}{l}\text { - Randomized } \\
\text { Controlled design } \\
\text { - Outpatient clinic } \\
\text { orders of } 74 \\
\text { physicians in US }\end{array}$ & $\begin{array}{l}\text { - Patient sessions were } \\
\text { randomized using } \\
\text { computer software } \\
\text { - Because of attrition } \\
\text { and completion of } \\
\text { training, ultimately } 74 \\
\text { of the } 121 \text { physicians } \\
\text { enrolled fully } \\
\text { participated and were } \\
\text { included in analysis. }\end{array}$ & $\begin{array}{l}\text { A window with itemized } \\
\text { charges was displayed } \\
\text { while ordering tests and } \\
\text { medications for } \\
\text { physicians in the } \\
\text { intervention group. }\end{array}$ & $\begin{array}{l}\text { Patient testing charges were } \\
12.9 \% \text { lower per visit for } \\
\text { the intervention group. } \\
\text { Residents ordered } 15.3 \% \\
\text { fewer tests, resulting in a } \\
13.4 \% \text { reduction in } \\
\text { charges. Faculty members } \\
\text { ordered } 7.9 \% \text { fewer tests, } \\
\text { resulting in an } 11.2 \% \\
\text { reduction in charges. } \\
\text { Cost Reduction: Statistically } \\
\text { significant } \\
\text { Decrease in number of tests } \\
\text { ordered statistically } \\
\text { significant }\end{array}$ \\
\hline $\begin{array}{l}\text { Articles on Medication Choice } \\
\text { Hart, J. et al. Do Drug } \\
\text { Costs Hart, } \\
\text { J. et al. Do Drug Costs } \\
\text { Affect Physician } \\
\text { Prescription Decisions? } \\
\text { J Intern Med. 1997; } \\
\text { 241: 415-420. }\end{array}$ & $\begin{array}{l}\text { - Pre-Intervention, } \\
\text { Post-Intervention } \\
\text { design } \\
\text { - Survey study of } \\
60 \text { Israeli } \\
\text { hospitalists and } \\
\text { family physicians }\end{array}$ & $\begin{array}{l}\text { - Participants were } \\
\text { not made aware } \\
\text { of the purpose of } \\
\text { the study } \\
\text { - Participation and } \\
\text { attrition rates not } \\
\text { reported }\end{array}$ & $\begin{array}{l}\text { Questionnaire describing } \\
\text { patients with severe } \\
\text { and mild urinary tract } \\
\text { infection administered } \\
\text { to hospitalist and family } \\
\text { physicians, first without } \\
\text { medication price, then } \\
2 \text { months later with prices. }\end{array}$ & $\begin{array}{l}\text { Hospitalists' choice of } \\
\text { antibiotics was significantly } \\
\text { different and favored the less } \\
\text { expensive medication when } \\
\text { showed price. Family physicians } \\
\text { chose } \\
\text { the less expensive antibiotic } \\
\text { regardless of price display. } \\
\text { Cost reduction - statistical } \\
\text { significance not reported } \\
\text { Medication choice - differences } \\
\text { statistically significant }\end{array}$ \\
\hline $\begin{array}{l}\text { Horrow, J. C. et al. Price } \\
\text { Stickers } \\
\text { Do Not Alter Drug Usage. } \\
\text { Can J Anaesth. 1994; } 41 \\
\text { (11): 1047-1052. }\end{array}$ & $\begin{array}{l}\text { - Pre-Intervention, } \\
\text { Post-Intervention } \\
\text { design } \\
\text { - } 56 \text { faculty, resident } \\
\text { and nurse anesthetists } \\
\text { in American OR }\end{array}$ & $\begin{array}{l}\text { - Participants were not } \\
\text { made aware of the } \\
\text { purpose of the study }\end{array}$ & $\begin{array}{l}\text { Supermarket-style price } \\
\text { stickers were placed } \\
\text { on units of selected } \\
\text { pharmaceuticals. }\end{array}$ & $\begin{array}{l}\text { No major differences in the } \\
\text { number of general } \\
\text { anesthetics per week. } \\
\text { Cost reduction - not statistically } \\
\text { significant } \\
\text { Medication choice - usage of } \\
2 / 11 \text { anesthetics measured were } \\
\text { statistically significantly different. }\end{array}$ \\
\hline
\end{tabular}


Table 2. (continued)

\begin{tabular}{|c|c|c|c|c|}
\hline Article citation & Study details & Bias considerations & Intervention & Results \\
\hline $\begin{array}{l}\text { Hux, J. E., Naylor, C. D. } \\
\text { Drug Prices and Third } \\
\text { Party Payment: Do They } \\
\text { Influence Medication } \\
\text { Selection? Pharmaco } \\
\text { Economics. 1994; } 5 \\
\text { (4): 343-350. }\end{array}$ & $\begin{array}{l}\text { - Randomized } \\
\text { Controlled design } \\
\text { - Mailed survey } \\
\text { collected from } \\
\text { 686 Canadian } \\
\text { PCPs }\end{array}$ & $\begin{array}{l}\text { - Questionnaires were } \\
\text { randomized at mailing } \\
\text { - } 71 \% \text { response rate } \\
\text { - No significant } \\
\text { differences in demo } \\
\text { graphics of participants }\end{array}$ & $\begin{array}{l}\text { Questionnaire describing } \\
\text { a COPD patient randomized } \\
\text { to four different scenarios: } \\
\text { (A) full insurance coverage } \\
\text { and prices of drugs listed, } \\
\text { (B) full insurance coverage } \\
\text { and prices not listed, (C) } \\
\text { prices listed but no insur } \\
\text { ance coverage, (D) neither } \\
\text { prices nor coverage. }\end{array}$ & $\begin{array}{l}\text { When prices were omitted, } 38 \% \\
\text { of physicians prescribed the } \\
\text { expensive option, vs. } 18 \% \text { when } \\
\text { coverage and prices were disclosed. } \\
\text { If it was disclosed that patient had } \\
\text { no drug coverage benefit, } 8 \% \\
\text { prescribed expensive antibiotic. } \\
\text { Cost reduction - n/a } \\
\text { Medication choice - statistically } \\
\text { significantly different }\end{array}$ \\
\hline $\begin{array}{l}\text { Lin, Y-C., Miller, S.R. } \\
\text { The Impact of Price } \\
\text { Labeling of Muscle } \\
\text { Relaxants on Cost } \\
\text { Consciousness among } \\
\text { Anesthesiologists. J Clin } \\
\text { Anesth. 1998; 10: } \\
\text { 401-403. }\end{array}$ & $\begin{array}{l}\text { - Pre-Intervention, } \\
\text { Post-Intervention } \\
\text { design } \\
\text { - 20,389 vs. } 20,538 \\
\text { cases in } \\
\text { American OR }\end{array}$ & $\begin{array}{l}\text { - Intervention and } \\
\text { Control periods } \\
\text { were seasonally } \\
\text { the same } \\
\text { - Participants were } \\
\text { informed of the } \\
\text { purpose of the study }\end{array}$ & $\begin{array}{l}\text { Subjects underwent an } \\
\text { educational session } \\
\text { about less expensive } \\
\text { muscle relaxants. The } \\
\text { price labels were placed } \\
\text { on vial caps of all muscle } \\
\text { relaxants for } 1 \text { year. }\end{array}$ & $\begin{array}{l}\text { Expenditures for the less costly } \\
\text { pancuronium increased while } \\
\text { expenditures for vecuronium } \\
\text { and atracurium decreased. } \\
\text { Total expenditure on muscle } \\
\text { relaxants decreased } 12.5 \% \text {, } \\
\text { saving } \$ 47,311 \text {. } \\
\text { Cost reduction - statistically } \\
\text { significant } \\
\text { Medication choice - not reported }\end{array}$ \\
\hline $\begin{array}{l}\text { McNitt, J. D. et al. } \\
\text { Long-Term Pharmaceutical } \\
\text { Cost Reduction Using } \\
\text { a Data Management } \\
\text { System. Anesth Analg. } \\
\text { 1998: 87: 837-842. }\end{array}$ & $\begin{array}{l}\text { - Pre-Intervention, } \\
\text { Post-Intervention } \\
\text { design } \\
\text { - 4,530 vs. } 10,600 \\
\text { anesthesia records } \\
\text { from American OR }\end{array}$ & $\begin{array}{l}\text { - Participants were } \\
\text { informed of the } \\
\text { purpose of the study }\end{array}$ & $\begin{array}{l}\text { Cost lists were placed in } \\
\text { each operating room, the } \\
\text { anesthesia satellite } \\
\text { pharmacy, and the } \\
\text { anesthesia lounge. } \\
\text { Providers were encouraged } \\
\text { to use less costly drugs. } \\
\text { Every 2-3 months, the } \\
\text { patterns of use and cost were } \\
\text { presented to the department. }\end{array}$ & $\begin{array}{l}\text { Costs decreased } \$ 30,000 \text { per month } \\
\text { and } \$ 32 \text { dollars per case. Quality } \\
\text { indicators did not change. } \\
\text { Cost reduction - reports percent } \\
\text { of change but not percentage } \\
\text { Medication choice - differences } \\
\text { statistically significant }\end{array}$ \\
\hline $\begin{array}{l}\text { Salman, H. et al. The } \\
\text { Effect of Drug Cost } \\
\text { on Hypertension } \\
\text { Treatment Decision. } \\
\text { Public Health. } \\
\text { 1999; 133: 243-246. }\end{array}$ & $\begin{array}{l}\text { - Pre-Intervention, } \\
\text { Post-Intervention } \\
\text { design } \\
\text { - Survey study of } \\
60 \text { Israeli hospitalist } \\
\text { and family } \\
\text { physicians }\end{array}$ & $\begin{array}{l}\text { - Participants were } \\
\text { not made aware of } \\
\text { the purpose of } \\
\text { the study } \\
\text { - Participation and } \\
\text { attrition rates not } \\
\text { reported }\end{array}$ & $\begin{array}{l}\text { Physicians were given two } \\
\text { hypothetical cases about the } \\
\text { treatment of hypertensive } \\
\text { patients. The first time, } \\
\text { prices were not displayed } \\
\text { on the questionnaire. Two } \\
\text { months later, the same } \\
\text { scenario was presented, } \\
\text { but with cost of medication } \\
\text { displayed. }\end{array}$ & $\begin{array}{l}\text { The second questionnaire showed } \\
60 \% \text { of the family physicians } \\
\text { and } 87 \% \text { of the hospital } \\
\text { physicians opted to prescribe the } \\
\text { less expensive drug combination. } \\
\text { Cost reduction }-\mathrm{n} / \mathrm{a} \\
\text { Medication choice - differences } \\
\text { were statistically significant }\end{array}$ \\
\hline
\end{tabular}

intervention arm were statically significant and resulted in $10 \%$ decrease in fees. ${ }^{14}$ Tierney et al. found that there were $14.9 \%$ fewer tests ordered and that testing charges were $12.9 \%$ lower per visit during the intervention period. ${ }^{19}$ Ellemdin et al. found a $27 \%$ reduction in mean cost per admitted patient. ${ }^{13}$ Hampers et al. noted a $27 \%$ decrease in charges during the intervention period compared with the control period. ${ }^{15}$

Effects on Medication Choice. Interestingly, all three clinically based interventions looking at medication choice came from the anesthesiology literature. ${ }^{20-22}$ All three studies were preintervention vs. post-intervention designs. Two interventions involved supermarket-style price stickers on medications ${ }^{20,21}$; one intervention used lists of drug costs. ${ }^{22}$ One study focused on muscle relaxants alone, ${ }^{21}$ whereas two included a broader spectrum of medications used in the operating room setting. ${ }^{20,22}$ Two interventions included an educational component. ${ }^{20,21}$

Two of three studies found a significant reduction in total medication expenditures; one study did not. Lin et al. reported a shift in muscle relaxant choice towards the less costly option that resulted in a total expenditure decrease of $12.5 \%{ }^{21}$ McNitt reported that the average savings after the intervention was $\$ 32 /$ case. $^{22}$ Both Lin et al. and McNitt et al. reported that
PACU and SICU admissions, as proxies for quality of care, were not increased due to medication choice..$^{21,22}$

\section{Surveys and Simulation Studies}

Of the five studies that looked at physician decisions in surveys or simulated settings, two looked at test ordering and three looked at medication ordering.

Effects on Radiology and Laboratory Test Ordering. Cummings et al. and Rudy et al. presented resident physicians with clinical scenarios and randomized surveyees to have charge information included in the portion of the survey in which the workup for the clinical scenario was assessed. Both studies noted a decrease in ordering when charge information was presented. Cummings et al. found that the cost of tests ordered for each hypothetical patient was $31.1 \%$ lower when price information was provided. ${ }^{23}$ The design of this study did include a minimum work-up for each scenario required to preserve quality of care. Both intervention and control groups met that standard. ${ }^{23}$ Rudy et al. found that residents with access to charge data spent less on tests $(\$ 1,297$ versus $\$ 2,205)$, but also had lower "appropriateness" scores, meaning that the quality of the care was impacted by the modified test ordering. ${ }^{24}$ 
Effects on Medication Choice. The three survey studies that examined medication choice surveyed non-US physicians about management of urinary tract infections, ${ }^{25}$ chronic obstructive pulmonary disease, ${ }^{26}$ and hypertension. ${ }^{27} \mathrm{Hux}$ et al. surveyed primary care physicians in Canada in which participants were randomized to receive information on drug prices and/or patient insurance coverage, or no information. Each surveyee was provided with a clinical scenario and asked about his/her choice of medication and management. ${ }^{26}$ Hart et al. and Salman et al. surveyed physicians in Israel with scenarios involving urinary tract infections and hypertensive patients, respectively. ${ }^{25,27}$ In both studies, participants received an initial survey that did not include price information. Two months later, participants received the same survey with price information. The differences in medication choice were compared.

Hux et al. found that the percentage of physicians prescribing the expensive antibiotic option dropped from 38 to $18 \%$ when insurance coverage and prices were disclosed. ${ }^{26}$ Hart et al. and Salman et al. both reported statistically significant differences in medication choices after prices were disclosed. Physicians surveyed prescribed the less expensive antibiotic $56 \%$ initially, compared to $83 \%$ when price was disclosed in Hart et al.. ${ }^{25}$ In Salman et al., cost disclosure prompted $57 \%$ of family practice physicians and $87 \%$ of hospitalists surveyed to choose the less expensive medication, both of which conferred statistically significant $p$ values. ${ }^{27}$

\section{CONCLUSIONS}

In this systematic review of charge transparency interventions, we found that having real-time access to charges changed ordering and prescribing behavior in the majority of studies. Of the clinically based interventions looking at laboratory and radiology ordering, seven of the nine studies reported statistically significant cost reduction when charges were displayed. Interestingly, of the six studies that reported differences in the number of tests ordered, only three reported a statistically significant decrease in the number of tests ordered. This may reflect that awareness of cost may lead a practitioner to order a less expensive test rather than fewer tests.

The clinically based interventions that focused on medication choice again trended towards a decrease in cost when currency amounts were displayed on medication - two of the three reported statistically significant reduction. All three survey studies also showed a trend towards choosing less expensive medication options when price was displayed, though these were hypothetical situations.

It is worth noting that the two studies with non-significant findings of the clinically based studies examined ordering patterns for radiology tests. Bates et al. reported a decrease in laboratory ordering, though not of statistical significance, and no difference in the ordering of radiology when price was displayed. ${ }^{11}$ Durand et al. only focused on radiology ordering, randomizing the various modalities that could be ordered, and found no difference. ${ }^{12}$

There was considerable heterogeneity in the clinical setting, patient population (pediatric vs. adult), health care system (international vs. US), study design, and outcomes measured. The majority of interventions took place in the inpatient setting, with two studies based in emergency medicine. Tierney et al. stands alone as the one outpatient clinically based study included in this analysis. ${ }^{19}$ All of these studies were conducted at a single site. Even among the clinically based randomized controlled interventions, there were differences in design: Feldman et al. and Durand et al. randomized the tests themselves, whereas Bates et al. and Tierney et al. randomized the patient encounters.

\section{DISCUSSION}

To our knowledge, no other literature review has specifically looked at real-time charge display and its impact on physician practice patterns. While this synthesis of data from the literature points toward the potential of cost-savings when prices are displayed, it is unclear whether universal availability of a currency amount will have enough impact to significantly bend the cost curve on a system-wide or national level. Indeed, as several recent articles have pointed out, ${ }^{4,6}$ finding exact charges of tests and medication can be very challenging - the resources necessary to find and integrate this information in real time, may outweigh the savings gained.

Another unanswered question is whether changes in practice from charge display affect quality of care. While some studies did incorporate a quality metric, the majority did not. A primary concern of physicians modifying practice patterns is that the quality of patient care will be compromised. Clearly, this is an area for further study.

Bias is another consideration in synthesizing these data. As the intervention in question is one of transparency, blinding subjects and assessors to the intervention is not possible. Several papers disclose that subjects were not aware that they were being studied; others specifically included an educational component as part of the intervention. The danger of performance bias and detection bias is inherent to these interventions. Reporting bias is another consideration, though we are reassured that studies with both significant and statically insignificant results have been included in the literature. Another limitation to acknowledge is that our review may have not have captured all articles on this subject. Indeed, only articles from the medical literature were ultimately included. We used search terms and search engines with the hopes of finding studies from policy, economics, and lay literature, but no additional interventions were identified.

Were charge data to be more broadly adopted, a significant issue to consider is what charge the ordering practitioner should use. There is often great discrepancy in the currency amount among what a hospital or clinic charges, what an insurance company reimburses, what a patient pays, and the 
cost to the larger medical system. These studies do not address which of these costs a clinician should consider when making ordering decisions. Indeed, the source of the charge presented was not consistently reported in these studies.

Finally, the decreases in costs reported in these studies focus primarily on the cost-savings to the hospital or clinical provider. What remains to be seen is whether charge transparency decreases medical expense to the patient. Potentially, the doctor-patient relationship could benefit from increased transparency about medical costs, though this has yet to be established. There are growing calls for physicians to factor the financial consequences into their medical decisions. ${ }^{28-30}$ Charge data offers additional information for physicians to make the most educated decisions for a patient's care.

Acknowledgements: Dr. Bishop is supported by a National Institute On Aging Career Development Award (K23AG043499) and by funds provided to her as a Nanette Laitman Clinical Scholar in Public Health at Weill Cornell Medical College.

Conflict of interest: The authors declare that they do not have a conflict of interest.

\section{Disclaimers: None.}

Corresponding Author: Celine Goetz, MD; Division of Hospital Medicine, Department of Medicine Mount Sinai Hospital, 1 Gustave Levy Place, Box 1087, New York, NY 10029, USA (e-mail: celine.goetz@mountsinai.org).

\section{REFERENCES}

1. McCarthy D et al. Why not the best? Results from the National Scorecard on U.S. Health System Performance, 2011. 2011, The Commonwealth Fund Commission on a High Performance Health System.

2. World Development Indicators, The World Bank. Last Updated: 06 Nov 2014. Website: http://wdi.worldbank.org/table/2.15. Accessed 28 Nov 2014.

3. Graham JD, Potyk D, Raimi E. Hospitalists' awareness of patient charges associated with inpatient care. J Hosp Med. 2010;5(5):295-297.

4. Allan GM, Lexchin J. Physician awareness of diagnostic and nondrug therapeutic costs: a systematic review. Int $\mathrm{J}$ Technol Assess Health Care. 2008;24(2):158-165.

5. Kullgren JT, et al. A census of state health care price transparency websites. JAMA. 2003;309(23):2437-2438.

6. Sinaiko AD, et al. Increased price transparency in health care-challenges and potential effects. N Engl J Med. 2011;364(10):891-894.

7. Steinmetz A, et al. What does a hip replacement cost? JAMA Intern Med. 2013; 173(6):432-434.
8. Bernstein JRH, et al. Availability of consumer prices from Philadelphia area hospitals for common services: electrocardiograms vs. parking. JAMA Intern Med. 2014;174(2):292-293.

9. Ivers N, Jamtvedt G, Flottorp S, Young JM, Odgaard-Jensen J, French SD, O'Brien MA, Johansen M, Grimshaw J, Oxman AD. Audit and feedback: effects on professional practice and healthcare outcomes. Cochrane Database Syst Rev. 2012;13:16.

10. Axt-Adam P, van der Wouden MA, van der Does E. Influencing behavior of physicians ordering laboratory tests: a literature study. Med Care. 1993;31(9):784-794.

11. Bates DW, et al. Does the computerized display of charges affect inpatient ancillary test utilization? Arch Intern Med. 1997;157(21):2501-2508.

12. Durand DJ, et al. Provider cost transparency alone has no impact on inpatient imaging utilization. J Am Coll Radiol. 2013;10(2): 108-113.

13. Ellemdin S, Rheeder P, Soma P. Providing clinicians with information on laboratory test costs leads to reduction in hospital expenditure. S Afr Med J. 2011;101(10):746-748.

14. Feldman LS, et al. Impact of providing fee data on laboratory test ordering: a controlled clinical trial. JAMA Intern Med. 2013;173(10):903-908.

15. Hampers LC, et al. The effect of price information on test-ordering behavior and patient outcomes in a pediatric emergency department. Pediatrics. 1999;103(4 Pt 2):877-882.

16. Sachdeva RC, et al. Effects of availability of patient-related charges on practice patterns and cost containment in the pediatric intensive care unit. Crit Care Med. 1996;24(3):501-506.

17. Schilling UM. Cutting costs: the impact of price lists on the cost development at the emergency department. Eur J Emerg Med. 2010;17(6):337-339.

18. Seguin $\mathbf{P}$, et al. Effects of price information on test ordering in an intensive care unit. Intensive Care Med. 2002;28(3):332-335.

19. Tierney WM, Miller ME, McDonald CJ. The effect on test ordering of informing physicians of the charges for outpatient diagnostic tests. N Engl J Med. 1990;322(21):1499-1504.

20. Horrow JC, Rosenberg H. Price stickers do not alter drug usage. Can J Anaesth. 1994;41(11): 1047-1052.

21. Lin YC, Miller SR. The impact of price labeling of muscle relaxants on cost consciousness among anesthesiologists. J Clin Anesth. 1998;10(5):401-403.

22. McNitt JD, Bode ET, Nelson RE. Long-term pharmaceutical cost reduction using a data management system. Anesth Analg. 1998;87(4):837-842.

23. Cummings KM, et al. The effects of price information on physicians' testordering behavior. Ordering of diagnostic tests. Med Care. 1982;20(3):293-301.

24. Rudy DW, et al. A pilot study assessing the influences of charge data and group process on diagnostic test ordering by residents. Acad Med. 2001;76(6):635-637.

25. Hart J, et al. Do drug costs affect physicians' prescription decisions? J Intern Med. 1997;241(5):415-420.

26. Hux JE, Naylor CD. Drug prices and third party payment: do they influence medication selection? Pharmacoeconomics. 1994;5(4):343-350.

27. Salman $\mathbf{H}$, et al. The effect of drug cost on hypertension treatment decision. Publ Health. 1999;133:243-246.

28. Kwok J, Jones B. Unnecessary repeat requesting of tests: an audit in a government hospital immunology laboratory. J Clin Pathol. 2005;58(5):457-462.

29. May TA, et al. Reducing unnecessary inpatient laboratory testing in a teaching hospital. Am J Clin Pathol. 2006;126(2):200-206.

30. Novich M, Gillis L, Tauber AI. The laboratory test justified. An effective means to reduce routine laboratory testing. Am J Clin Pathol. 1985;84(6):756-759. 